\title{
PERFORMANCE CALCULATION OF A MULTI-STAGE AXIAL COMPRESSOR THROUGH A SEMI-EMPIRICAL MODELLING FRAMEWORK
}

\author{
Samuel Cruz-Manzo \\ University of Lincoln \\ scruzmanzo@lincoln.ac.uk \\ Lincoln, United Kingdom
}

\author{
Vili Panov \\ Siemens Industrial Turbomachinery \\ vili.panov@siemens.com \\ Lincoln, United Kingdom
}

\author{
Senthil Krishnababu \\ Siemens Industrial Turbomachinery \\ senthil.krishnababu@siemens.com \\ Lincoln, United Kingdom
}

\begin{abstract}
In this study, calculation of the performance of an axial compressor from a twin-shaft industrial gas turbine (IGT) is carried out through a semi-empirical modelling framework. The modelling framework considers a flow analysis represented through theoretical air velocity diagrams in the multi-stage axial compressor. Design parameters, such as blade angle and annulus geometry across the different stages comprising the multi-stage axial compressor, are considered for the development of the modelling framework. Stagewise data generated through a High-Fidelity Design Tool (HFDT) during compressor simulation are considered for the validation of the modelling framework. The data comprise flow rate, rotational speed, inlet guide vane (IGV) position, variable stator guide vanes (VGVs) position, temperature, and pressure ratio across the different stages. The results demonstrate that it is possible for the developed modelling framework to predict the increase of pressure and temperature across the different stages of the compressor in a way similar to HFDT. Capability of the modelling framework to predict the effect of IGV position outside its nominal schedule on stage compressor performance is demonstrated through the construction of an overall compressor map and individual stage characteristic maps. In addition, the effect of a hypothetical VGV mechanism malfunction situation on stagewise pressure ratio is predicted. The effect of fouling which impacts the turning of the air passing through the blades in the axial compressor that lead to compressor performance deterioration is also predicted. This study couples the fundamental principles of axial compressors and a semiempirical modelling to include more physics into the low fidelity modelling framework.
\end{abstract}

\section{INTRODUCTION}

Industrial gas turbines (IGT) are used in different industrial applications such as power generators and mechanical drivers. Multi-stage axial compressors are commonly used in IGTs and consist of a series of stages. Each stage comprises a set of rotor blades followed by a set of stator blades. The kinetic energy of air is increased by the rotor blades and the stator blades transform the kinetic energy of air into static pressure. A variation of the angle of the stator blades across the different stages of an axial compressor ensures correct air diffusion at different operating conditions (Razak, 2007).

Figure 1 shows a simplified mechanism to change the angle of the variable stator guide vanes (VGVs) using a single actuator. The closure and opening of VGVs are a function of the demanded inlet guide vane (IGV) position. The IGV position is commonly scheduled with respect to gas generator speed (GGS) of gas turbines (Muir et al., 2009).

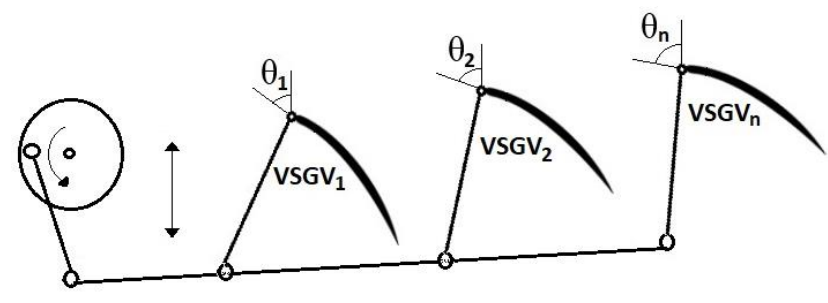

Figure 1 VGV mechanism in axial compressor

Studies in the literature have reported multi-stage axial compressor models. (Tournier and El-Genk, 2010) developed a multi-stage axial compressor for high temperature nuclear 
reactor power plants with Closed Brayton Cycle for energy conversion. The model considered a mean-line through-flow analysis for free-vortex flow along the blades. Pressure losses were considered and the effects of work coefficient, flow coefficient and stage reaction on the design and performance of compressor were investigated. The model was validated with measurements of the helium compressor of the GTHTR300, HTR power plant. A one-dimensional, stage-bystage, axial compressor performance model was reported by (Johnson, 1991). The model considered turbomachinery fundamentals and pressure losses through the compressor blade rows. The model required airfoil geometry and designpoint performance parameters. It was demonstrated to be very useful to predict the off-design performance pressure of large utility gas turbine power systems. A new model to predict changes of tip clearance of an 11-stage axial compressor was proposed by (Dong et al., 2014). The model considered firstprinciples to model the thermal and mechanical effects of engine operating conditions on the compressor subcomponents. The model was validated using General Electric's E3 engine experimental results. The results demonstrated the losses and blockages near the casing due to tip clearance flows of the 11-stage compressor. (Alm-Eldien et al., 2013) reported two computer programs for the design and optimization of a multi-stage axial compressor. The programs considered axi-symmetric flow properties such as pressure, temperature and velocity along streamlines at the entry and exit of each stage. In addition, the programs considered analytical relationships between isentropic efficiency of the axial-flow compressor, the flow coefficient, the work coefficient, degree of reaction and various design parameters. The results provided general guidance for the optimum design of the axial-flow compressors. Other studies in the literature have reported various multi-stage compressor models to study the effect of variable geometry on compressor performance. The performance of a multi-stage axial compressor was analysed through a numerical method reported by (Song et al., 2001). The method considered the variation in the IGV and VGV in the compressor performance. The results showed the reduction of air flow and pressure ratio result from the closure of stator vanes. A stage-stacking method to estimate the variable geometry effects across the different stages of an axial compressor was reported by (Muir et al., 2009). The results showed that an increase of one degree in the variable geometry position (closure of VGV) resulted in $3 \%$ reduction in air flow, $4 \%$ reduction in fuel flow and a $5 \%$ power loss. (Tsalavoutas et al., 2000) reported failures in the VGV system of a multi-stage axial compressor of an IGT. The authors examined faults located at different stages and different characteristics using adaptive performance modelling and monitoring the circumferential distribution of the turbine exit temperature. Interestingly, the authors reported that the turbine exit temperature distributions are affected by the presence of compressor faults.

In a previous study (Cruz-Manzo, et al., 2017), a thermodynamic model to predict the performance of a twinshaft IGT at different operating conditions was developed. The model consisted of a lumped axial compressor model comprising overall performance maps for flow, pressure and efficiency. However, the model could only estimate the total pressure, temperature and flow discharged by the compressor. In the current study, a semi-empirical model, based on design parameters, compressor performance data set, and theoretical relations (velocity triangles for blade design) from an 11-stage axial compressor has been developed to predict the individual stage performance of the axial compressor. Stagewise pressure ratio and temperature collected through a High-Fidelity Design Tool (HFDT) during multi-stage axial compressor simulation have been considered to validate the developed modelling architecture. It is also possible to calculate the individual stage performance of the compressor through the construction of characteristic maps across the different stages. The availability of design parameters and real-world compressor performance data from the compressor manufacturer assisted the simplification of the modelling architecture compared with the other modelling architectures previously discussed. An additional test case in which the effect of IGV position outside of its nominal schedule on stage performance was carried out. Further development of this modelling framework could provide an insight into the effect of different compressor failure $\backslash$ degradation modes (CruzManzo, et al., 2018) such as fouling and out of position VGV on different stages of the axial compressor.

\section{THEORETICAL BACKGROUND}

The power input to the axial compressor stage can be related to the stage velocity triangles. The velocity triangles are commonly applied to relate air diffusion with compressor design parameters. Fig. 2 shows the velocity diagrams for the rotor inlet and rotor outlet for a single stage of the compressor; $\alpha_{l}$ represents the angle of the air absolute velocity $C_{l}$ from the axial direction $C_{X}$ entering the rotor.
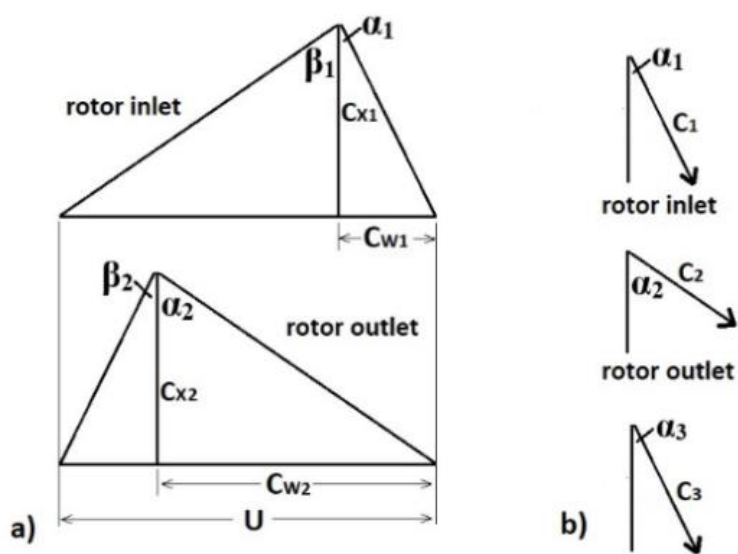

b)
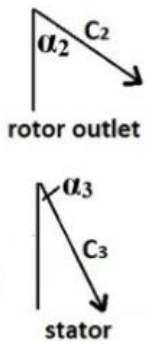

Figure 2 Velocity triangles for a single stage of the axial compressor, reproduced from Saravanamutto et al., 2009, a) velocities related to the rotor inlet and rotor outlet, b) air absolute velocities for rotor and stator

For the first stage $\alpha_{l}$ can be taken to represent the IGV angle if flow deviation is ignored for simplicity. Similarly, $\beta_{1}$ 
can be taken to represent the rotor blade inlet angle; $\alpha_{2}$ is the angle of the absolute air velocity $C_{2}$ leaving the rotor, $\beta_{2}$ represents the rotor blade outlet angle, and $\alpha_{3}$ is the angle of the absolute air velocity diffusing through the stator. $U$ represents the rotor tangential velocity (blade velocity). Analysis of these velocity triangles utilising the trigonometric relations and the thermodynamic principles can provide the temperature and pressure rise across the compressor stages. Further information about the relation between the velocity triangles and thermodynamic principles can be found in Saravanamutto et al., 2009.

The difference in total temperature between the outlet and inlet of the compressor stage is defined as:

with

$$
\Delta \mathrm{T}_{2-1}=\frac{\mathrm{U} \lambda \mathrm{C}_{\mathrm{X}}}{\mathrm{C}_{\mathrm{P}}}\left(\tan \alpha_{2}-\tan \alpha_{1}\right)
$$

$$
\mathrm{U}=\mathrm{r} \omega \text {. }
$$

where $U$ is the blade velocity, $r$ is the tip radius, and $\omega$ is the angular velocity; and

$$
\mathrm{C}_{\mathrm{X}}=\frac{\dot{\mathrm{m}}}{\rho A} \text {. }
$$

$C_{X}$ is the air axial velocity, $\dot{m}$ is the air flow rate, $\rho$ is the air density, and $A$ is the annulus area. $\lambda$ is the work-done factor and accounts for the reduction in work capacity across the compressor axial stage (Razak, 2007). The work-done factor $\lambda$ can be defined as the ratio between the actual and ideal work that absorb work capacity in the stage. It can also be defined as the loss in work capacity due to variation of air velocity across the stages. The parameter $\lambda$ can be derived from the Euler turbine equation. (Saravanamutto et al., 2009).

The pressure ratio through the stage can be defined as:

$$
\tan \alpha_{2}-\tan \alpha_{1}=\frac{\mathrm{C}_{\mathrm{P}} \mathrm{T}_{\mathrm{in}}}{\eta \mathrm{U} \lambda \mathrm{C}_{\mathrm{X}}}\left[\mathrm{P}_{\mathrm{R}}^{\frac{\gamma-1}{\gamma}}-1\right] .
$$

where $\eta$ is the stage efficiency, and $T_{i n}$ is the total inlet temperature entering the stage.

\section{OVERALL MODELLING STRUCTURE}

Compressor design parameters, certain flow parameters across the compressor and the equations defined in the previous section are required to develop the semi-empirical modelling architecture. The design parameters comprise annulus area $A$, tip radius $r$ and blade angles $\alpha, \beta$ across the different stages of the compressor which are provided by the compressor manufacturer. The pressure ratio and temperature ratio for each stage were gathered during compressor simulation at different rotational speeds. These parameters were calculated using a HFDT. HFDT is a Siemens standard through flow analysis code which computes the performance of the multi-stage compressor as an axisymmetric model.

The demanded IGV angle has been scheduled with respect to compressor speed. The position (degrees) of VGVs is a function of IGV position.
The running line representing the overall performance of the compressor is shown in Fig. 3. It shows the total pressure ratio vs flow rate at different operational speeds. The pressure ratio and flow rate have been normalized with respect to the maximum pressure ratio and maximum flow rate values. The operational speed has been normalized with respect to the maximum speed $\omega_{\max }$.

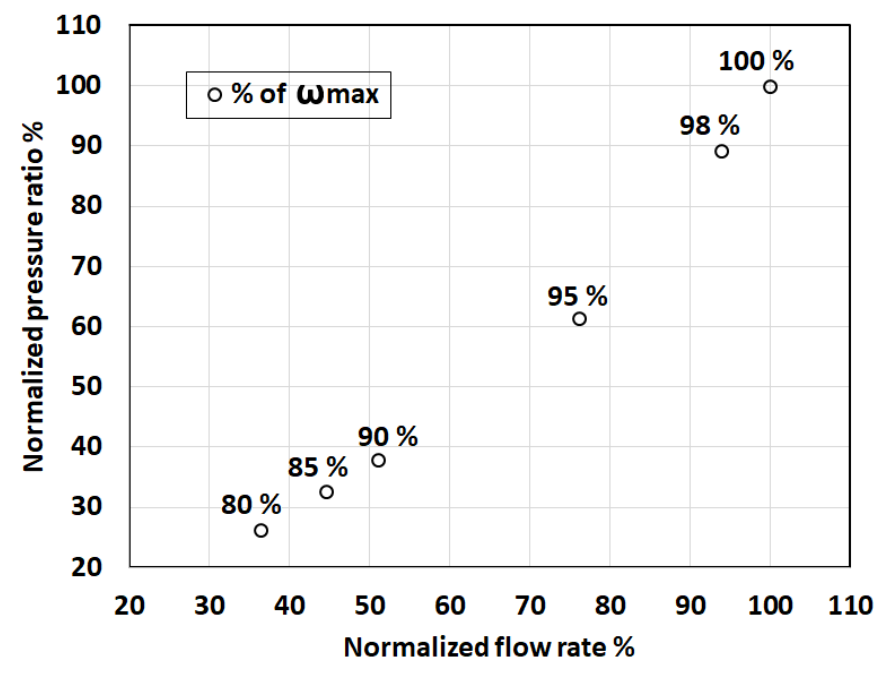

Figure 3 Normalized total pressure ratio vs normalized flow rate at different speeds

Some theoretical assumptions have been considered to facilitate the development of the modelling architecture:

- $\quad$ The stage velocity triangles shown in Fig. 2 are only related to the rotor blade air angles, considering that the power is absorbed in the rotor and the stator transforms kinetic energy to an increase in static pressure. Under this condition, the static enthalpy rise in the rotor exceeds the enthalpy rise in the stator for the static pressure rise.

- The accessibility of design parameters and compressor performance data across the stages allowed the estimation of the work-done factor $\lambda$ which accounts for the reduction in work capacity across the stages. This parameter could be considered to represent losses across the compressor. This simplified the mathematics without the need to implement individual equations (Tournier and ElGenk, 2010; Johnson, 1991) to predict various losses in the axial compressor.

- No fluid friction in the stator which decreases the pressure is considered.

$\alpha_{1}$ in the first stage corresponds to the IGV angle. The angle $\alpha_{3}$ of the absolute velocity in the axial direction diffusing through the stator as shown in Fig. $2 b$ is equal to the angle $\alpha_{I}$ entering the rotor from the subsequent stage.

A graphic user interface (GUI) has been constructed in Matlab® environment to execute the semi-empirical modelling framework represented in Fig. 4. 
The GUI requires the rotational speed $\omega$, flow rate $\dot{m}$, and ambient temperature $T_{a m b}$ as inputs. The rotational speed and flow rate are for a selected operating point from the compressor performance map shown in Fig. 3. The outputs from the GUI are the pressure ratio $P_{R}$, and temperature $T_{\text {out }}$ across the different stages of the axial compressor. The GUI can interpolate and extrapolate values of pressure ratio and temperature ratio for each stage not provided by the HFDT. This will be demonstrated later in the construction of the compressor map. The data representing stagewise temperature and pressure collected from the HFDT are initially required for the calculation of thermodynamic parameters such as air density $\rho$, heat capacity $C_{P}$, and ratio between heat capacities $\gamma$ across the different stages. The flow rate $\dot{m}$, annulus area $A$, and density $\rho$ allow the calculation of the axial flow velocity $C_{X}$ defined in Eq. 3. The IGV position $\alpha_{1}$ as a function of compressor speed and the VGVs position as a function of IGV position are also fed into the solver.

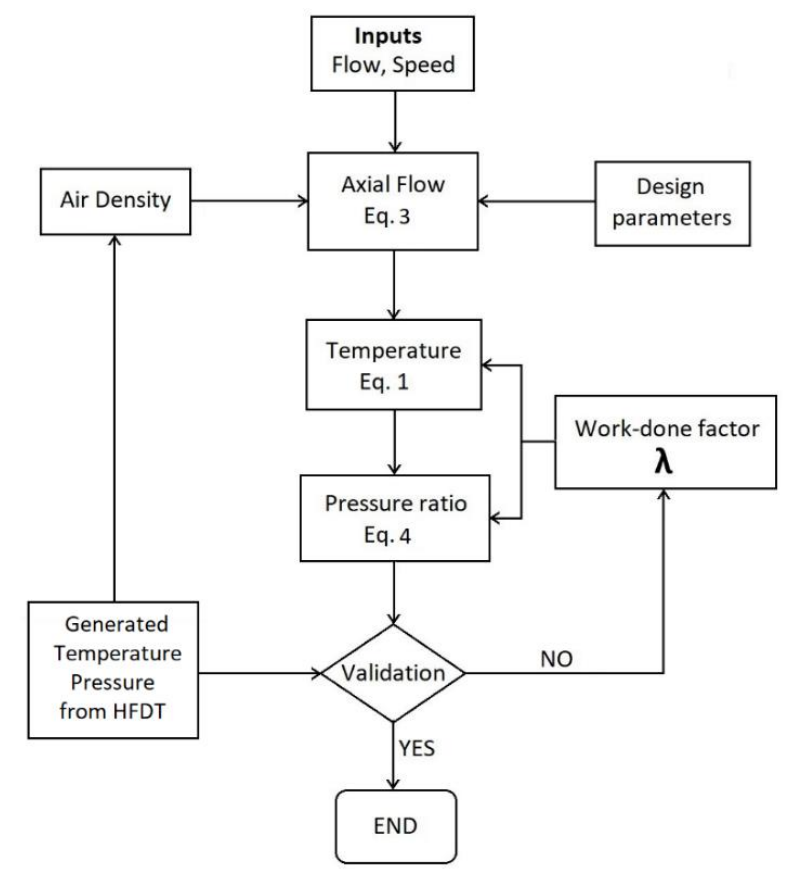

Figure 4 Semi-empirical modelling architecture

The modelling architecture is executed in two parts. It consists of an optimization process and a calculation process. The following steps comprise the optimization process.

1. A nonlinear least-squares solver defined in the Optimization toolbox of Matlab® software is used to tune $\lambda$ (the work-done factor) to match the stagewise data for temperature and pressure ratios with the temperature and pressure ratios predicted by Eqs. 1 and 4 respectively.

2. Eqs. 1 and 4 comprise the parameter $\lambda$; therefore, the optimization procedure reduces the error between HFDT stagewise data and simulated data from the GUI and calculates a value of $\lambda$ using Eq. 1 and Eq. 4 respectively.

The second process during the execution of the modelling architecture considers the calculation process to predict stagewise pressure and temperature. The following steps comprise the calculation process:

1. The resulting average value of $\lambda$ estimated during the optimization process is considered to calculate stagewise pressure and temperature using Eqs. 1 and 4.

2. It is not only possible to predict stagewise pressure and temperature from HFDT, but also to interpolate and extrapolate stagewise pressure and temperature not provided by HFDT.

\section{VALIDATION}

The flowchart shown in Fig. 4 described the process that has been used for the validation of the pressure ratio and temperature predicted by the model. Three different speeds (viz., $80 \%, 95 \%$, and $100 \%$ of the maximum speed $\omega_{\max }$ ) have been selected to demonstrate the prediction capability of the model. The considered data for the model validation, correspond to three different speeds of the running line shown in Fig. 3. The running line represents the compressor performance at nominal conditions, e.g. the IGV position follows its nominal schedule. In addition, compressor performance data when the IGV position is outside its nominal schedule have been considered for the model validation. The HFDT temperature and pressure data are compared with the temperature and pressure ratio predicted by the model at these speeds in Figs. 5-9. It is seen that the model can accurately predict the distribution of temperature and pressure ratio across the different stages. The data shown in Figs. 5-9 have been normalised with respect to the maximum stagewise pressure ratio and temperature value. Fig. 5 clearly shows smaller pressure ratio in the front stages of the compressor compared to the rest of the compressor. This can be attributed to the lower air diffusion restricted by the closure of the VGVs in these stages.

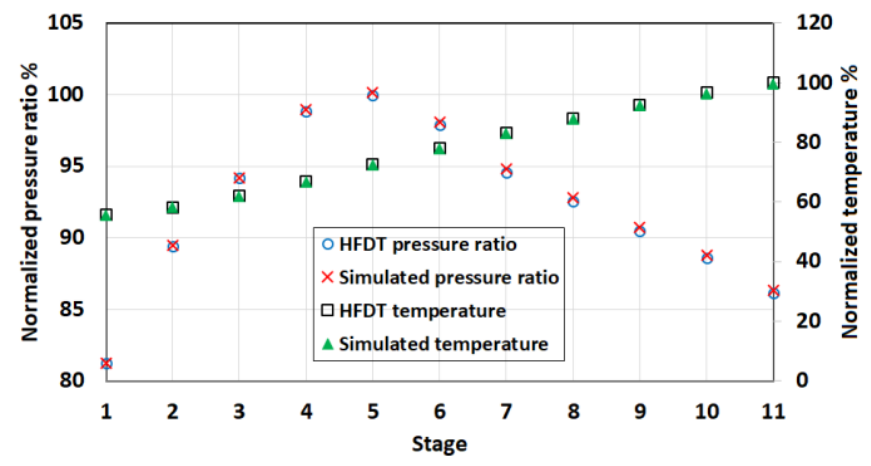

Figure 5 Comparison between HFDT data and simulated data at $80 \%$ of $\omega_{\max }$

An increase in pressure ratio during the front stages of the compressor is achieved with increasing compressor speed at $95 \%$ of $\omega_{\max }$, as shown in Fig. 6.

Fig. 7 shows a decrease in pressure ratio from the front stages to the rear stages at $\omega_{\max }$. This can be attributed to increase in the stage inlet temperature as the air flow diffuses through the compressor. 


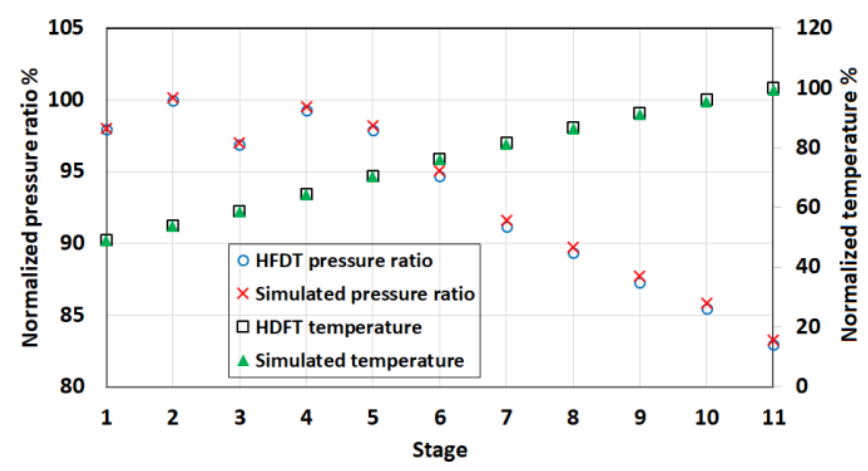

Figure 6 Comparison between HFDT data and simulated data at $95 \%$ of $\omega_{\max }$

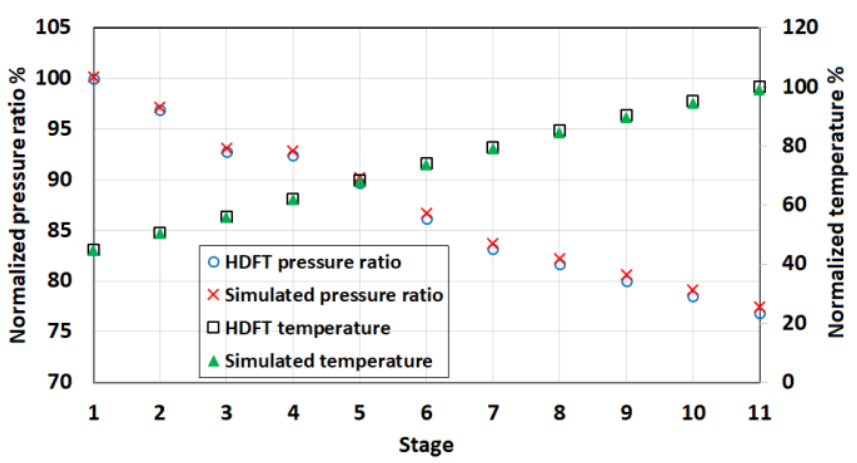

Figure 7 Comparison between HFDT data and simulated data at $\omega_{\max }$.

These results show that the model can predict the HFDT pressure ratio and temperature across the different stages of the compressor at various speeds.

In addition, compressor performance when IGV is hypothetically put into a position outside its nominal schedule have been considered for the validation. A positive IGV offset is related to an increase of the IGV position outside its nominal schedule and a negative IGV offset is a decrease of the IGV position outside its nominal schedule.

Fig. 8 shows the comparison between HFDT stagewise pressure ratio data and simulated data for +5 IGV offset position.

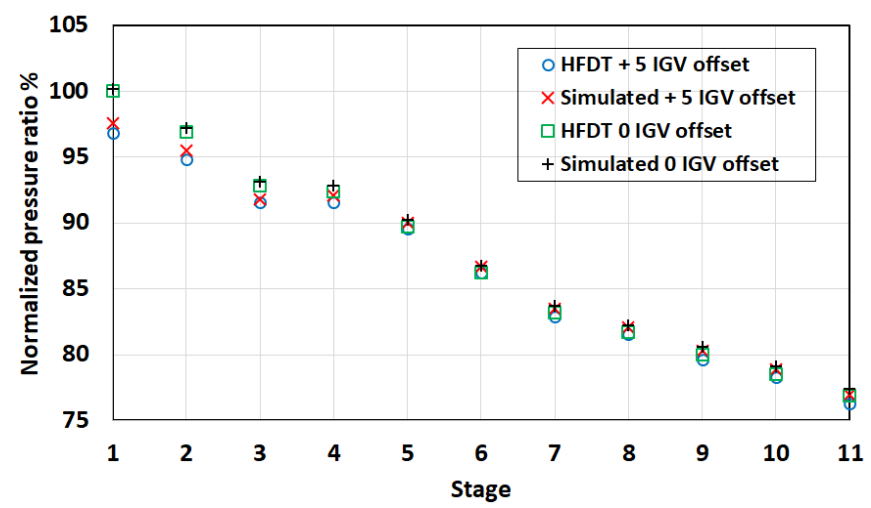

Figure 8 Comparison between HFDT data and simulated data for pressure ratio at $\omega_{\max }$ with +5 IGV Offset and no IGV Offset.
Stagewise pressure ratio for the nominal IGV case is also shown in Fig. 8 for comparison. Under the condition at +5 IGV offset the compressor inlet flow decreased by $4 \%$ with respect to the flow at nominal conditions (no IGV offset). The values of pressure ratio have been normalized with respect to the maximum value of pressure ratio collected from HFDT at nominal conditions.

Fig. 9 shows a similar comparison between HFDT pressure ratio data and simulated data at $\omega_{\max }$ and with a -5 IGV offset. The compressor inlet flow increased by $3 \%$ with respect to the flow at nominal conditions. The normalized stagewise pressure ratio for the case with -5 IGV offset increases compared to that with no IGV offset.

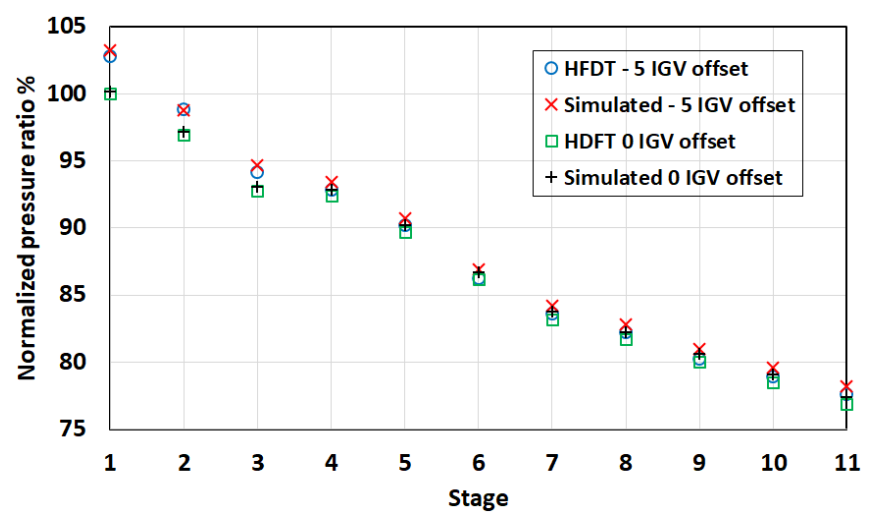

Figure 9 Comparison between HFDT data and simulated data for pressure at $\omega_{\max }$ with -5 IGV Offset and no IGV Offset.

The results shown in Figs. 5-9 demonstrate that the model can predict stagewise pressure (and temperature, not shown in this paper) at different operating speed and different IGV offset position.

TABLE I. \% Error between HFDT and simulated pressure ratio

\begin{tabular}{|c|c|c|c|c|c|}
\hline Stage & $\begin{array}{c}80 \% \\
\omega_{\max }\end{array}$ & $\begin{array}{c}95 \% \\
\omega_{\max }\end{array}$ & $\omega_{\max }$ & $\begin{array}{c}\omega_{\max } \\
5 \text { IGV } \\
\text { offset }\end{array}$ & $\begin{array}{c}\omega_{\max } \\
\text { 5 IGV } \\
\text { offset }\end{array}$ \\
\hline 1 & 0.000 & 0.083 & 0.179 & 0.714 & 0.398 \\
\hline 2 & 0.026 & 0.140 & 0.239 & 0.639 & 0.027 \\
\hline 3 & 0.057 & 0.175 & 0.306 & 0.231 & 0.477 \\
\hline 4 & 0.117 & 0.281 & 0.443 & 0.612 & 0.611 \\
\hline 5 & 0.170 & 0.367 & 0.537 & 0.494 & 0.519 \\
\hline 6 & 0.205 & 0.410 & 0.589 & 0.528 & 0.794 \\
\hline 7 & 0.220 & 0.434 & 0.626 & 0.691 & 0.701 \\
\hline 8 & 0.242 & 0.468 & 0.678 & 0.630 & 0.633 \\
\hline 9 & 0.247 & 0.478 & 0.708 & 0.843 & 0.885 \\
\hline 10 & 0.244 & 0.472 & 0.721 & 0.749 & 0.842 \\
\hline 11 & 0.215 & 0.416 & 0.711 & 0.828 & 0.755 \\
\hline
\end{tabular}

The estimated absolute value of the percentage error between stagewise data and simulated data for pressure ratio 
is shown in Table I. The error increases from front to rear of the compressor and with increase in speed. The error can be attributed to the errors in calculation of thermodynamic parameters such as air density $\rho$, heat capacity $C_{P}$, and ratio between heat capacities $\gamma$ using HFDT stagewise pressure and temperature.

The maximum error between stagewise data simulated data is less than $1 \%$. Although it is considered that the parameter $\lambda$ accounted for losses across the stages, the error could be reduced if other mechanisms are considered such as fluid friction in the stator which decreases the pressure rise or if blade-profile pressure losses are also considered (Lieblein, 1959). The error between HFDT stagewise data and simulated data for temperature is not shown, but the temperature error resulted to be less than the pressure ratio error.

\section{RESULTS}

In the previous section, validation for stagewise temperature and pressure rise was carried out at different operational speeds for +5 and -5 IGV offset position. In this section, a prediction of the overall performance map at \pm 7.5 and \pm 2.5 offsets from nominal IGV positions at maximum compressor speed is presented.

Fig. 10 shows the resulting compressor map predicted by the developed model at different IGV offset position at $\omega_{\max }$ for a selected point above and below the $100 \%$ pressure ratio point. The speed lines coined as "prediction" represent the prediction by the model by interpolating and extrapolating the parameter $\lambda$ in the modelling architecture.

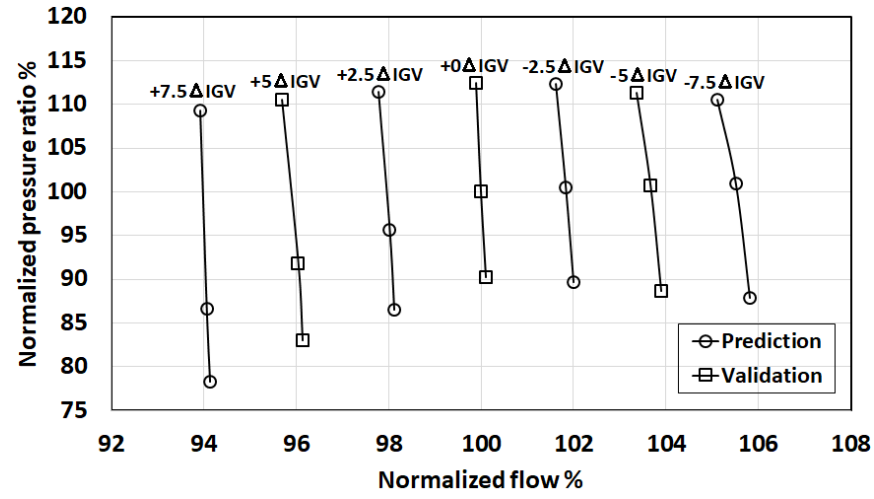

Figure 10 Overall compressor map at different IGV offset position.

The parameter $\lambda$ plays an important role and is the main parameter during the prediction of the compressor performance, as shown in Fig. 4. The parameter $\lambda$ is considered to represent all the losses across the compressor barring those mentioned above. This modelling process will simplify the construction of the overall compressor map at different operating conditions and provides flexibility in the integration of the 1-D model with gas turbine models (CruzManzo, et al., 2018) comprising 0-D model components.

Stage performance maps can be constructed considering pressure and flow coefficients as reported by (Song et al., 2001):

$$
\varphi=\frac{C_{X}}{U} \text {. }
$$

where Eq. 5 is defined as the flow coefficient and Eq. 6 is the pressure coefficient.

$$
\psi=\frac{C_{P} T_{i n}\left(\operatorname{Pr}^{\frac{\gamma-1}{\gamma}}-1\right)}{U^{2}} .
$$

The nomenclature of Eqs. 5 and 6 has been described in the theoretical section. The stage characteristic maps have been constructed from the overall compressor map shown in Fig. 10 considering nominal condition (no offset) and IGV offset value of +5 . Figs. 11 and 12 show the constructed stagecharacteristic maps for 0 , and +5 IGV offset.

Fig. 11 represents stage performance with no IGV offset position. Each mark reflects the individual stage performance that corresponds to the normalized total pressure ratio from the overall compressor map with no IGV offset. The maximum pressure coefficient $\psi$ in each stage is related to the normalized total pressure ratio with value of $113 \%$ from the overall compressor map (0 IGV offset). The other pressure ratio coefficients in each stage are related to the total pressure ratio with value of $100 \%$ and $90 \%$ from the overall compressor map (Fig. 10) respectively. The last stage (11) presents the minimum pressure coefficient $\psi$. This can be attributed to the minimum stagewise pressure ratio within the last stage. This can be observed in the stagewise pressure ratio profile from Fig. 7.

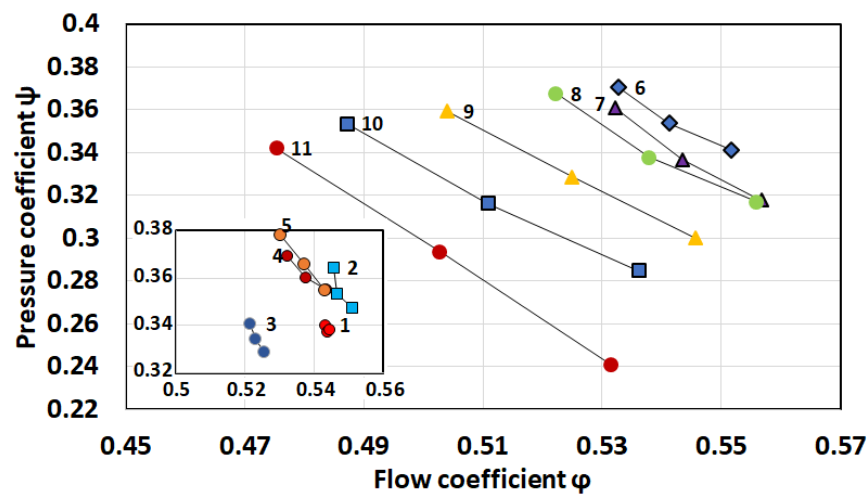

Figure 11 Stage characteristic map at $\omega_{\max }$ with no IGV Offset.

The stage-characteristic maps at $\omega_{\max }$ with $+5 \mathrm{IGV}$ offset are shown in Fig. 12.

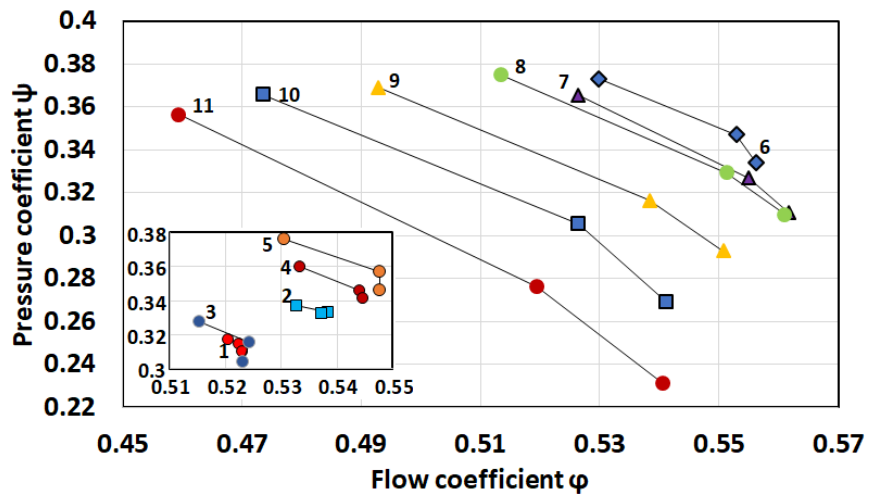

Figure 12 Stage characteristic map at $\omega_{\max }$ with +5 IGV Offset. 
Change in the slope of the stage performance lines with increasing pressure coefficient $\psi$ and reducing flow coefficient $\varphi$ can be seen in Fig. 12. This change in the slope can be related to the movement of the stages towards its stability limit.

\section{VGV MALFUNCTION}

The modelling framework developed in this study can also be used to predict compressor performance during malfunction in the VGV mechanism and during fouling conditions. Effects on compressor performance due to failure in the VGV mechanism and fouling conditions will be investigated and validated in future work. A demonstration of the prediction of compressor performance due to mal-function in of the VGV mechanism is presented here. As previously mentioned, in normal situations the position of the VGVs follows a nominal schedule. For the purpose of this demonstration it is assumed that the last two VGVs do not follow the nominal schedule when compressor is accelerated from $90 \%$ to $100 \%$ speed.

Figure 13 shows a decrease in pressure ratio as a consequence of a failure in the last two VGVs which failed to follow the nominal speed schedule during the transition from $90 \%$ to $100 \%$ compressor speed. Noting that the pressure ratio at nominal conditions corresponds to the predicted stagewise pressure ratio previously shown in Fig. 7. The failure in the VGV mechanism yielded a reduction in the overall total pressure ratio of $6 \%$ with respect to nominal conditions. The analysis shown in Fig. 13 has been carried out at steady-state conditions. The effect of VGV failure on compressor performance during dynamic conditions needs further investigation, as this effect may drift the stage characteristic performance map closer to the unstable condition during dynamic operation in certain conditions.

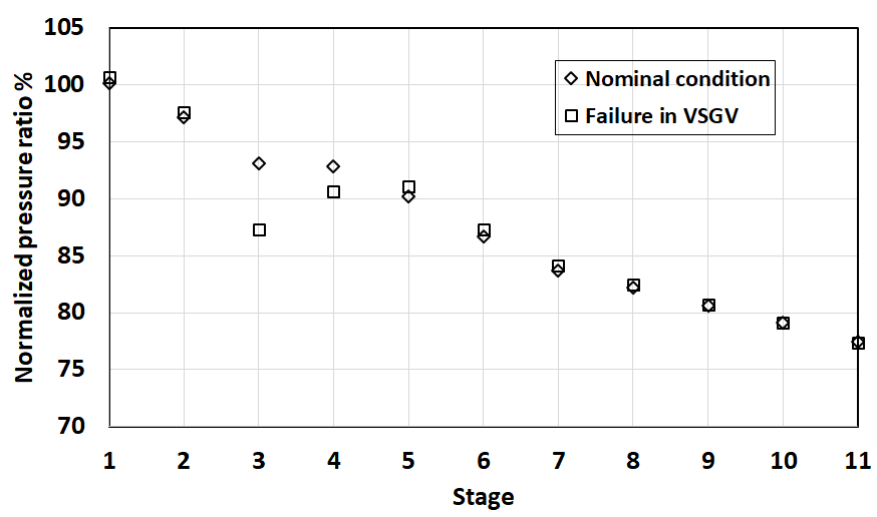

Figure 13. Normalized pressure ratio at $\boldsymbol{\omega}_{\max }$ considering nominal conditions and failure in the VGV mechanism: VGVs 3 and 4 mal-function.

\section{COMPRESSOR FOULING}

The developed modelling framework can be applied to predict the effect of fouling on compressor performance. Fouling condition in axial compressors is attributed to airborne particles (dust, mineral particles, etc.) attached to the blades which decreases its performance. These particles can increase the surface roughness of the blades and impact the turning of the air passing through the blades in the axial compressor. It is possible to represent the velocity triangles of axial compressor at fouling conditions (Song et al., 2005).

In Fig. 14 dotted line represents the change in velocity diagrams during fouling conditions (reduction in axial velocity and hence reduction in flow discharged by the compressor). Thus, during fouling conditions, there is a decrease in axial velocity entering and leaving the rotor $C x_{1}$ and $C x_{2}$ 'respectively and hence a reduction of the rotor blade inlet and outlet angles $\beta_{1}^{\prime}$ and $\beta_{2}{ }^{\prime}$. a)

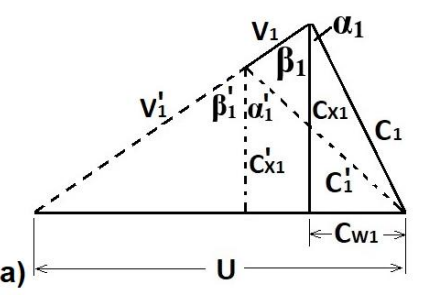

b)

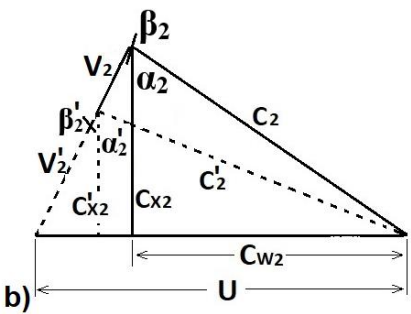

Figure 14. Velocity triangles at fouling conditions, a) rotor inlet, b) rotor outlet.

It is possible to represent fouling by considering a deviation angle, $\delta$. This yields a change in angles $\alpha_{1}$ and $\alpha_{2}$ as shown in Eqs. 7 and 8.

$$
\begin{aligned}
& \alpha_{1}^{\prime}=\alpha_{1}+\delta \\
& \alpha_{2}^{\prime}=\alpha_{2}-\delta
\end{aligned}
$$

Eqs. 1 and 4 can now be expressed in terms of $\alpha^{\prime}$ instead of $\alpha$. Note that the value of the fouling factor $\delta$ will depend on the degree of fouling (airborne particles) or roughness in the blades of the compressor and may be different across the different stages of the axial compressor. The fouling factor is a time dependent parameter and increases in time from nominal conditions (at clean conditions) to a high fouling condition. (Mal'tsev and Shakhov, 1989) reported air deviation angles due to roughness on the blades of the axial compressor. A deviation angle of 3 degree for a relative roughness of $0.01 \mathrm{k}_{\mathrm{s}} / \mathrm{c}$ was reported, where $k_{s}$ is the surface roughness $(\mu \mathrm{m})$, and $c$ is the chord length $(\mathrm{m})$. (Tarabrin et al., 1998) studied the performance of a two-shaft Frame 5 engine and concluded that mainly the front stages of the compressor are more affected by fouling. Similar conclusion was reported by (Song et al., 2005). In this study, the deviation in flow due to roughness on the blade surfaces represented by the fouling factor $\delta$ for the first five stages of the compressor was taken to be $3\left(\alpha_{1}^{\prime}=\alpha_{1}+3\right)$. Thereafter, the deviation angle $\delta$ is reduced to 2.25 for the rest of the stages. These values are considered to be representative of a fouled compressor after many hours of compressor operation, (Cruz-Manzo, et al., 2018).

Fig. 15 shows the simulation results from which a reduction in stagewise pressure ratio due to fouling is seen. Note that the pressure ratio at nominal conditions corresponds to the simulated stagewise pressure ratio previously shown in Fig. 7. The reduction in pressure ratio is more noticeable in the first four stages (front-end) of the compressor as a lower air deviation angle in the remaining stages of the compressor 
was used. The analysis shown in Fig. 15 has been carried out at steady-state conditions. In future the dynamic reduction of stagewise performance in the compressor under fouled conditions will be studied. In a previous study (Cruz-Manzo, et al., 2018) simulation of compressor fouling conditions, using a Simulink engine model comprising a 0-D compressor model and real-world validation data from engines operated as mechanical drivers and power generators, has been carried out. In future work, the 1-D compressor modelling architecture developed in this study will be incorporated into a Simulink engine model to predict and validate reduction in engine performance attributed to compressor fouling conditions.

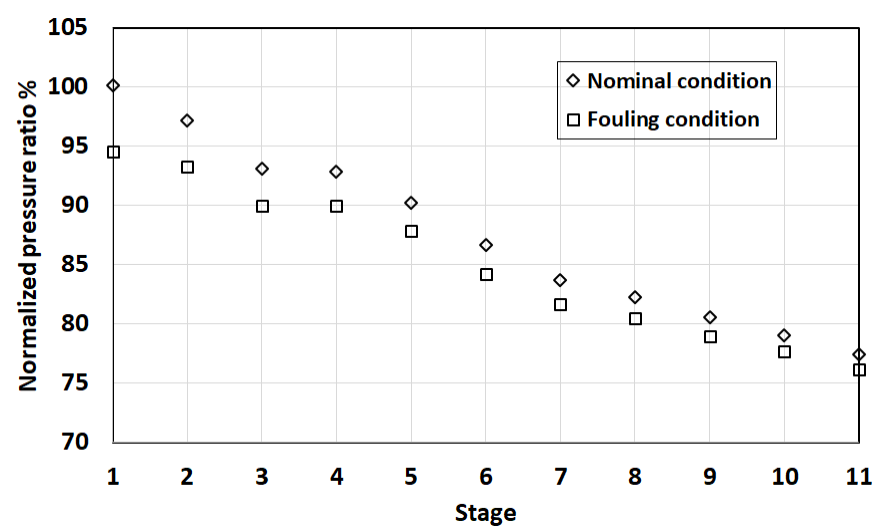

Figure 15. Normalized pressure ratio at $\omega_{\max }$ considering nominal conditions and fouling conditions.

\section{DISCUSSION AND RECOMMENDATION}

The prediction of stage-characteristics in axial compressors could provide valuable information during the dynamic operation of an axial compressor in the field. For instance, during the dynamic transition between two operating conditions a given stage may drift close to its stability limit which could cause instability of the whole compressor during the dynamic operation particularly at low speeds. In this paper the ability of the developed modelling architecture in predicting the effect of IGV mal-function and the effect of fouling on stage performance were demonstrated. The developed modelling architecture can predict the performance of the compressor attributed to changes in blade geometry and blade angle across the different stages of the compressor. Therefore, any abnormal condition during compressor operation such as icing, erosion, etc. which may change the geometry and angle of the blades across the different stages of the compressor could be predicted by the multi-stage compressor model developed from this study. This developed modelling architecture could provide flexibility for the integration of the 1-D compressor model within the whole gas turbine models (Cruz-Manzo, et al., 2017; Panov, 2009) commonly comprising 0-D individual component models. In a previous study (Cruz-Manzo, et al., 2018) the change in air temperature and air pressure discharged by an axial compressor during fouling conditions was simulated through a Simulink 0-D gas turbine model. The simulation results were validated against real measurements and the analysis consisted

of an empirical modification of flow capacity and efficiency from the compressor. Deposition of dirt and dust particles on the compressor blades increase surface roughness on the blades and changes the velocity triangles representing the compressor blades (Tarabrin et al., 1998). In a future work, the developed 1-D modelling architecture from this study will be implemented in the 0-D engine model from the previous study (Cruz-Manzo, et al., 2018) to simulate engine performance during compressor fouling conditions by varying the velocity triangles at different compressor stages.

\section{CONCLUSION}

A semi-empirical modelling framework comprising theoretical relations, design parameters, and stagewise data of a multi-stage axial compressor has been developed. The modelling framework considers theoretical relations represented by air velocity diagrams in the multi-stage axial compressor. Design parameters, such as blade angle and annulus geometry across the different stages comprising the multi-stage axial compressor, were also considered for the development of the modelling framework. In addition, stagewise temperature and pressure data collected through a HFDT during compressor simulation were required for the development of the semi-empirical modelling framework. The stagewise data comprise flow, speed, IGV and VGVs position, temperature, and pressure ratio. The simulated data predicted by the model were compared with HFDT stagewise data and the results demonstrated that it is possible to predict the increase of pressure and temperature through the different stages of the compressor using the developed model. The IGV position depends on speed and can differ for raising and falling speed. Different IGV position changes the angle of the VGVs through a linear motion mechanism. A change in IGV position outside the nominal speed schedule impacts the performance of the multi-stage axial compressor. Capability of the developed modelling architecture in predicting the effect of IGV mal-function and the effect of fouling on stage performance were demonstrated. In future work, the developed modelling architecture will be validated at lower speeds. The developed theoretical framework could provide a better prediction of dynamic compressor performance especially during engine low load operation in which the compressor running line may be closer to its stability limit at certain conditions.

\section{NOMENCLATURE}

List of symbols

A

$C$

$C_{P}$

$C_{X}$

$C_{W}$

$\dot{m}$

$P_{R}$

$r$

$U$

T annulus area $\left(\mathrm{m}^{2}\right)$

air absolute velocity $(\mathrm{m} / \mathrm{s})$

heat capacity at constant pressure $(\mathrm{J} / \mathrm{kgK})$

air axial velocity $(\mathrm{m} / \mathrm{s})$

air tangential velocity $(\mathrm{m} / \mathrm{s})$

air flow rate $(\mathrm{kg} / \mathrm{s})$

pressure ratio (dimensionless)

radius of the annulus (m)

blade velocity $(\mathrm{m} / \mathrm{s})$

temperature $(\mathrm{K})$ 
air angle from the axial direction rotor blade inlet/outlet angle (rad) work-done factor (dimensionless) isentropic efficiency (dimensionless) air flow deviation (rad)

\section{ACKNOWLEDGMENT}

This research was supported by Siemens Industrial Turbomachinery, Lincoln, U.K.

\section{REFERENCES}

Alm-Eldien A. M., Gawad A. F. A., Hafaz G., Kreim M. G. A. E. (2013). Design and Optimization of a Multi-Stage Axial-Flow Compressor, Eleventh International Conference of Fluid Dynamics, Alexandria, Egypt, December 19-21.

Cruz-Manzo, S., Panov, V., Zhang, Y. (2018). Gas Path Fault and Degradation Modelling in Twin-Shaft Gas Turbines. Machines, 6, 43.

Cruz-Manzo, S., Panov, V., Zhang, Y., Latimer, A., Agbonzikilo, F. (2017). A thermodynamic transient model for performance analysis of a twin shaft Industrial Gas Turbine. In Proceedings of the ASME Turbo Expo 2017, Charlotte, NC, USA, June 26-30.

Dong Y., Xinqian Z., Qiushi L. (2014). An 11-stage axial compressor performance simulation considering the change of tip clearance in different operating conditions, Proc IMechE Part A: J. Power and Energy, 228, 614-625.

Johnson M. S. (1991). One-Dimensional, Stage-by-Stage, Axial Compressor Performance Model, ASME 1991 International Gas Turbine and Aeroengine Congress and Exposition, Orlando, Florida, USA, June 3-6.

Lieblein S. (1959). Loss and stall analysis in compressor cascades. J Basic Eng., 81, 387-400.

Mal'tsev Y. N., Shakov V.G. (1989). Influence of roughness of deposits in compressor cascade on flow lag angle (English translation), Izvestiya VUZ, Aviatsionnaya Tekhnika, 32, 80-82.

Muir, D.E.; Saravanamutto, H.I.H.; Marshall, D.J. (2009). Health Monitoring of Variable Geometry Gas Turbines for Canadian Navy. J. Eng. Gas Turbines Power, 111, 244-250.

Panov V. (2009). GasTurbolib-Simulink Library for Gas Turbine Engine Modelling, Proceedings of ASME Turbo Expo 2009, GT2009, June 8-12, Orlando, Florida, USA.

Razak V. A. (2007). "Industrial Gas Turbines Performance and Operability", Woodhead Publishing Limited.

Saravanamuttoo H., Rogers G., Cohen H., Straznicky P. (2009). Gas Turbine Theory, 6th Edition, Prencice Hall.

Song, T.W., Kim, T.S., Kim, J.H., Ro, S.T. (2001). Performance prediction of axial flow compressors using stage characteristics and simultaneous calculation of interstage parameters. Proc. Inst. Mech. Eng. 215, 89-98.

Song T. W., Sohn J. L., Ro S. T. (2005). Predictions of the performance degradation of industrial gas turbines due to compressor fouling, 16th International symposium of transport phenomena, ISTP-16, Prague.
Tarabrin, A. P., Bodrov, A. I., Schurovsky, V. A., Stalder, J-P. (1998). Influence of Axial Compressor Fouling on Gas Turbine Unit Performance Based on Different Schemes and with Different Initial Parameters, ASME International Gas Turbine and Aeroengine Congress, Stockholm Sweden, June 2-5, ASME paper No. 98-GT-416.

Tournier J.-M., El-Genk M. S. (2010). Axial flow, multistage turbine and compressor models, Energy Conversion and Management, 51, 16-29.

Tsalavoutas, A., Mathioudakis, K., Stamatis, A., Smith, M. (2000). Identifying Faults in the Variable Geometry System of a Gas Turbine Compressor. J. Turbomach. 123, 3339. 